\title{
Adherence to a healthy lifestyle and all- cause and cause-specific mortality in Chinese adults: a 10-year prospective study of 0.5 million people
}

Nanbo Zhu' ${ }^{1}$ Canqing Yu ${ }^{1}$ Yu Guo ${ }^{2}$, Zheng Bian², Yuting Han ${ }^{1}$, Ling Yang ${ }^{3,4}$, Yiping Chen ${ }^{3,4}$, Huaidong Du, Huimei $\mathrm{Li}^{5}$, Fang Liu${ }^{6}$, Junshi Chen ${ }^{7}$, Zhengming Chen ${ }^{4}$, Jun $\mathrm{Lv}^{1,8,9^{*}} \mathrm{D}$, Liming $\mathrm{Li}^{1}$ and on behalf of the China Kadoorie Biobank Collaborative Group

\begin{abstract}
Background: Adherence to a healthy lifestyle is associated with substantially lower risks of mortality from all causes, cardiovascular diseases, and cancer in white populations. However, little is known about the health benefits among non-white populations. Also, no previous studies have focused on respiratory disease mortality in both white and non-white populations. We assessed the relationships between a combination of healthy lifestyle factors and multiple death outcomes in Chinese adults.

Methods: This study included 487,198 adults aged 30-79 years from the China Kadoorie Biobank without heart disease, stroke, and cancer at study enrolment. We defined five healthy lifestyle factors as never smoking or smoking cessation not due to illness; non-daily drinking or moderate alcohol drinking; median or higher level of physical activity; a diet rich in vegetables, fruits, legumes and fish, and limited in red meat; a body mass index of 18.5 to $27.9 \mathrm{~kg} / \mathrm{m}^{2}$ and a waist circumference $<90 \mathrm{~cm}$ (men)/85 cm (women). Cox regression was used to produce adjusted hazard ratios (HRs) relating these healthy lifestyle factors to all-cause and cause-specific mortality.

Results: During a median follow-up of 10.2 years (IQR 9.2-11.1), we documented 37,845 deaths. After multivariable adjustment, the number of healthy lifestyle factors exhibited almost inverse linear relationships with the risks of allcause and cause-specific mortality. Compared with participants without any healthy factors, the hazard ratio of participants with five healthy factors was 0.32 [95\% confidence interval (Cl): 0.28, 0.37] for all-cause mortality. The corresponding HRs in specific cause of death were 0.42 (95\% Cl: $0.26,0.67)$ for ischaemic heart disease, 0.21 (95\% Cl: $0.09,0.49)$ for ischaemic stroke, 0.37 ( $95 \%$ Cl: $0.22,0.60$ ) for haemorrhage stroke, 0.36 ( $95 \%$ Cl: $0.29,0.45)$ for cancer, 0.26 (95\% Cl: $0.14,0.48)$ for respiratory diseases, and 0.29 (95\% Cl: $0.22,0.39)$ for other causes. Theoretically, 38.5\% ( $95 \%$ Cl: $33.0,43.8 \%$ ) of all-cause mortality was attributable to nonadherence to a healthy lifestyle, and the proportions of preventable deaths through lifestyle modification ranged from 26.9 to $47.9 \%$ for cause-specific mortality.

(Continued on next page)
\end{abstract}

\footnotetext{
* Correspondence: epi.lvjun@vip.163.com

'Department of Epidemiology and Biostatistics, School of Public Health,

Peking University Health Science Centre, 38 Xueyuan Road, Beijing 100191,

China

${ }^{8}$ Key Laboratory of Molecular Cardiovascular Sciences (Peking University),

Ministry of Education, Beijing, China

Full list of author information is available at the end of the article
}

(c) The Author(s). 2019 Open Access This article is distributed under the terms of the Creative Commons Attribution 4.0 International License (http://creativecommons.org/licenses/by/4.0/), which permits unrestricted use, distribution, and reproduction in any medium, provided you give appropriate credit to the original author(s) and the source, provide a link to the Creative Commons license, and indicate if changes were made. The Creative Commons Public Domain Dedication waiver (http://creativecommons.org/publicdomain/zero/1.0/) applies to the data made available in this article, unless otherwise stated. 
(Continued from previous page)

Conclusions: Adherence to a healthy lifestyle was associated with substantially lower risks of all-cause, cardiovascular, respiratory, and cancer mortality in Chinese adults. Promotion of a healthy lifestyle may considerably reduce the burden of non-communicable diseases in China.

Keywords: Healthy lifestyle, Mortality, Non-communicable diseases, Cohort study, Chinese

\section{Introduction}

Non-communicable diseases (NCDs), once considered "diseases of affluence", have now encroached on low- and middle-income countries, where three quarters of worldwide NCD deaths occurred [1]. In China, more than eight out of ten deaths are caused by NCDs, with ischaemic heart disease (IHD, 15\%), cerebrovascular disease (21\%), cancer $(23 \%)$, and chronic respiratory diseases $(12 \%)$ posing the greatest threat [2]. With the development of drug therapy for NCDs, more people are living with chronic conditions. However, escalating treatment expenditure is becoming unbearable, especially for poorer countries [3]. Were we to still rely heavily on treatment, global health resources would be inadequate to tackle the growing epidemic. Hence, population-wide primary prevention targeted at reducing exposure of risk factors should be the overarching priority for the response to this global crisis.

The combined impact of lifestyle factors, including tobacco smoking, excessive alcohol intake, physical inactivity, unhealthy diet, and obesity, on mortality has been prospectively evaluated mostly in western populations [4-6]. A meta-analysis showed that a combination of at least four healthy lifestyle factors lowered the all-cause mortality risk by $66 \%$ [7]. Some studies also demonstrated considerably lower risks of cardiovascular and cancer mortality ascribed to a healthy lifestyle [8-14]. However, no prior studies have focused on respiratory disease mortality, which is thought to be closely linked to infection, occupational hazards, and ambient and household air pollution in low- and middleincome countries. It is uncertain to what extent respiratory disease mortality is attributable to unhealthy lifestyle in this population. Also, there exist racial/ethnic disparities in disease subtype composition. For example, East Asia, notably China, has higher stroke incidence and a higher proportion of haemorrhagic stroke [15]. The effectiveness of lifestyle modification strategy in NCD prevention among non-white populations remains to be investigated.

The present study used data from the China Kadoorie Biobank (CKB) study, a nationwide prospective cohort of 0.5 million adults. We aimed to examine the associations of a combination of healthy lifestyle factors with the risks of all-cause and cause-specific mortality, and estimate the proportion of deaths that could theoretically be prevented through lifestyle modification during a 10-year period. Specifically, we sought to gain an insight into the possibly different impacts on specific causes of death.

\section{Methods \\ Study population}

The CKB cohort was established during 2004-2008 in 10 geographically diverse areas of China, including five urban and five rural areas. Detailed descriptions of the CKB cohort have been previously published $[16,17]$. All participants provided written informed consent, completed interviewer-administered questionnaires, and had physical measurements taken. Trained staff entered baseline information directly into a laptop-based data entry system developed with built-in functions to avoid missing items and minimise logic errors during the interview. The study received approval from the Ethical Review Committee of the Chinese Centre for Disease Control and Prevention (Beijing, China) and the Oxford Tropical Research Ethics Committee, University of Oxford (UK).

Overall, a total of 512,714 participants 30-79 years of age were eligible for inclusion. We excluded participants with self-reported clinician diagnosis of heart disease $(n=15,472)$, stroke $(n=8884)$, or cancer $(n=2578)$, and those who had missing data for body mass index (BMI, $n=2$ ), leaving 487,198 participants for the current analyses. For death from respiratory diseases, we additionally excluded participants with chronic obstructive pulmonary disease (COPD, $n=37,055)$, which was ascertained based on clinical diagnosis of emphysema or bronchitis prior to the baseline survey and pulmonary function test at the time of enrolment [18], leaving 452, 657 participants for this part of analysis.

\section{Assessment of lifestyle factors}

Baseline questionnaire and physical measurement assessed the lifestyle factors of interest. Inquiry on tobacco smoking included smoking status (never, former, or current smoker); ever smokers were then asked for the frequency, type, and amount of tobacco smoked per day, and former smokers were additionally asked for years since quitting and the reason for smoking cessation. Questions about alcohol intake included typical drinking frequency (never, occasionally, monthly, weekly, or daily); drinkers with the frequency of at least once a week were further asked for the type of alcoholic beverage drunk habitually, and the volume of alcohol drunk on a typical drinking day in the past 12 months.

Information on physical activity was obtained by asking participants about their usual type and duration of 
activities in each of the four domains (occupational, commuting, domestic, and leisure-time) in the past 12 months. We calculated the total physical activity level by multiplying the metabolic equivalent tasks (METs) value for each activity by hours spent on that activity per day and summing the MET-hours for all activities [19] (see Additional file 1: Supplementary Material S2 and S3). Habitual dietary intakes during the past year were assessed via a validated qualitative food frequency questionnaire (see Additional file 1: Supplementary Material S4 and S5), which covered 12 major food groups in China. Each food group was provided with five frequency categories to choose from (never/ rarely, monthly, 1-3 days/week, 4-6 days/week, or daily). Trained staff using calibrated instruments measured standing height, body weight, and circumferences of waist and hip. BMI was calculated as weight divided by height squared $\left(\mathrm{kg} / \mathrm{m}^{2}\right)$.

\section{Assessment of covariates}

Covariate information was obtained from the baseline survey as well, including sociodemographic characteristics, personal and family medical history, and women's reproductive information. Participants who reported at least one first-degree relative suffered from acute myocardial infarction, stroke, or cancer were considered as having a family history of corresponding disease. Prevalent hypertension was defined as measured systolic blood pressure $\geq 140 \mathrm{mmHg}$, or measured diastolic blood pressure $\geq 90 \mathrm{mmHg}$, or self-reported diagnosis of hypertension, or self-reported use of antihypertensive medication at baseline. Prevalent diabetes was defined as selfreported diabetes or screen-detected diabetes at baseline. Missing rates for menopausal status and three types of family history were $<1 \%$, and no other exposures or covariates contained missing values. The missing values were categorised into a "missing" group when analysing.

\section{Definition of healthy lifestyle}

We considered five lifestyle factors that have proved to be closely related to the risks of cardiovascular diseases (CVDs) and diabetes in our population [20, 21], namely, smoking, alcohol intake, physical activity, diet, and body shape. Lifestyle factors were deemed protective on the basis of previous studies, existing guidelines [22, 23], and their impacts on the present population. Healthy group regarding smoking behavior was defined in accordance with the recommendation that emphasizes the importance of not smoking and urges smoking cessation. We excluded those who had quit smoking because of illness, otherwise, it could lead to a misleadingly high risk in former smokers. The healthy group for alcohol intake comprised never-regular drinkers, weekly drinkers, and moderate daily drinkers (i.e., drinking $<30 \mathrm{~g}$ of pure alcohol in men and $<15 \mathrm{~g}$ in women per day).

For physical activity, groups were dichotomized based on the age- ( $<50$ years, $50-59$ years, and $\geq 60$ years $)$ and sex-specific median of total physical activity level, with the higher half constituting the healthy group. For diet, based on suggestions from the Chinese Dietary Guidelines [22] and in accordance with the previous findings [24], we considered five food groups, including fresh vegetables, fresh fruits, red meat, legumes, and fish. A simple diet score was created according to the criteria as follows: eating vegetables daily, eating fruits daily, eating red meat $1-6$ days per week, eating legumes $\geq 4$ days per week, eating fish $\geq 1$ day per week. For each food group, the participant who met the criterion received a score of 1 , and otherwise received a score of 0 . Therefore, the diet score ranged from 0 to 5 . Participants who scored 4 to 5 were classified to be in the healthy group. For body shape, both body weight and body fat were taken into account, as a reflection of energy balance [25]. Participants having a BMI between 18.5 and $27.9 \mathrm{~kg} / \mathrm{m}^{2}$ as well as a $\mathrm{WC}<90 \mathrm{~cm}$ in men and $<85 \mathrm{~cm}$ in women constituted the healthy group [23], emphasizing avoidance of extremely high or low weight and abdominal obesity.

The number of the healthy lifestyle factors was counted, with a range from 0 (unhealthiest) to 5 (healthiest).

\section{Ascertainment of deaths}

All ten study areas are covered by the Chinese Disease Surveillance Points system [26, 27], which provides cause-specific mortality data. Linkages with DSP death registries and local residential records, combined with annual active follow-up, were used to ascertain participants' vital status. All deaths were coded using the 10th International Classification of Diseases by trained staff, who were blinded to baseline information. The main outcomes in the analyses were all-cause mortality, as well as mortality resulting from IHD (I20-I25), ischaemic stroke (I63), haemorrhagic stroke (I61), cancer (C00C97), respiratory diseases (J00-J99), and all other causes.

\section{Statistical analysis}

Person-years at risk were calculated from the date of the baseline survey to the date of death, loss to followup, or December 31, 2016, whichever came first. We used stratified Cox proportional hazard models, with age as the underlying time scale, to estimate the hazard ratios (HRs) and 95\% confidence intervals (CIs) for the associations of individual and combined lifestyle factors with risks of all-cause and cause-specific mortality. The proportional hazards assumption was checked by comparing the HRs for the first and second half of the follow-up period. 
Multivariable models were stratified jointly by study area and age at baseline in 5-year intervals, and adjusted for sex, education, marital status, hip circumference, family histories of heart attack, stroke, or cancer, and menopausal status (only in women) at baseline. When analysing individual lifestyle factors, all the lifestyle factors were included simultaneously. We included hip circumference in the model because prior studies suggested adjustment for hip circumference may allow a more precise estimation of the detrimental effects of visceral adipose tissue which was measured by waist circumference [28]. Several sensitivity analyses were conducted to examine the robustness of our results: (1) further adjusting for potential confounders including socioeconomic status (household income and occupation), or metabolic risk factors (hypertension and diabetes); (2) excluding underweight participants (BMI $<18.5 \mathrm{~kg} / \mathrm{m}^{2}$ ); and (3) excluding deaths occurring during the first 2 years of follow-up.

To quantify the contribution of unhealthy lifestyle to the burden of disease, we calculated population attributable risk percent (PAR\%), which can be interpreted as the proportional reduction in population mortality that would have occurred during follow-up if all participants had adopted a healthy lifestyle. The multivariableadjusted PAR\% and 95 CIs were estimated using a previously proposed method [29]. As PAR\% is a populationspecific calculation that combines both relative risks and prevalence of risk factors, we further performed stratified analyses by sex, age, education, household income, residence, family history, and baseline status of hypertension and diabetes. In sensitivity analyses, we excluded ever smokers, underweight participants, and deaths that occurred during the first 2 years of follow-up.

The calculation of PAR\% was performed using SAS (version 9.4, SAS Institute Inc.), and all other statistical analyses were performed using Stata (version 15.0, StataCorp).

\section{Results}

Of the 487,198 participants, the mean age at baseline was 51.5 years (SD 10.5) and 287,958 (59.1\%) were women. Overall, the proportions of healthy group for each lifestyle factor were $70.6 \%$ for smoking, $92.9 \%$ for alcohol intake, $49.8 \%$ for physical activity, $8.5 \%$ for diet, and $71.1 \%$ for body weight and fat. Over $90 \%$ of participants had two to four healthy lifestyle factors, while only $2.1 \%$ had all five factors (Table 1 ). Women were more likely than men to adopt a healthy lifestyle, despite a slightly higher prevalence of obesity. With the increase in the number of healthy lifestyle factors, participants were younger, more educated, and less likely to be hypertensive or diabetic.
During a median follow-up of 10.2 years (a total of 4.8 million person-years), we documented 37,870 deaths, including 5116 from IHD, 1578 from ischaemic stroke, 4503 from haemorrhagic stroke, 12,620 from cancer, and 3655 from respiratory diseases (1833 remained after excluding participants with prevalent COPD at baseline). After adjustment for potential confounders, non-smoking, higher levels of physical activity, and higher diet score were associated with lower risk of all-cause mortality (Additional file 1: Table S1). For body shape, the lowest risk occurred in those with a BMI of $18.5-27.9 \mathrm{~kg} / \mathrm{m}^{2}$ and absence of abdominal obesity; both underweight and general and/or abdominal obesity were associated with higher risk. For alcohol intake, a J-shaped association was seen, with the lowest risk of allcause mortality for those consuming $1-15 \mathrm{~g}$ per day. In general, similar associations were observed for cardiovascular (Additional file 1: Table S2) and non-cardiovascular mortality (Additional file 1: Table S3), although with a few exceptions. For instance, light to moderate alcohol intake appeared to be more protective against IHD mortality, whereas heavy alcohol intake had more pronounced adverse effect on mortality from haemorrhagic stroke and cancer. Besides, the higher risk of cancer mortality was primarily seen among underweight participants, but that of stroke mortality was primarily seen among obese participants.

When healthy lifestyle factors were considered separately, all of them were associated with lower risk of allcause mortality (Table 2). However, some causes of death were not significantly associated with nonsmoking, non-excessive alcohol intake, or healthy dietary habits. When healthy lifestyle factors were combined, as shown in Fig. 1, the number of healthy factors exhibited almost inverse linear relationships with the risks of allcause and cause-specific mortality (all $P$ for linear trend $<0.001)$. In comparison with participants without any healthy factors, the adjusted HR $(95 \% \mathrm{CI})$ of participants who had five healthy factors was $0.32(0.28,0.37)$ for allcause mortality. The corresponding HRs in specific cause of death were $0.42(0.26,0.67)$ for IHD, $0.21(0.09$, $0.49)$ for ischaemic stroke, $0.37(0.22,0.60)$ for haemorrhagic stroke, $0.36(0.29,0.45)$ for cancer, $0.26(0.14$, $0.48)$ for respiratory diseases, and $0.29(0.22,0.39)$ for other causes. In the sensitivity analyses, further adjusting for potential confounders, and excluding underweight participants or deaths occurring in the first 2 years of follow-up did not substantially alter the risk estimates (Additional file 1: Table S4).

We calculated PAR\% for individual and combined lifestyle factors. With regard to all-cause mortality, the estimated PAR\% (95\% CI) was $10.6 \%(9.5,11.7 \%)$ for tobacco smoking, $1.3 \%(0.9,1.7 \%)$ for excessive alcohol intake, $13.9 \%(12.9,15.0 \%)$ for lack of physical activity, $11.8 \%(8.0,15.5 \%)$ for an unhealthy diet, and 


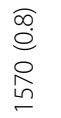

in

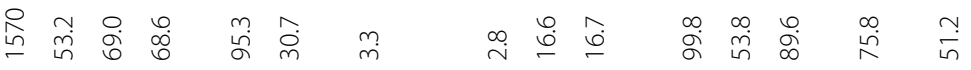

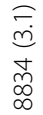

$\stackrel{\infty}{\Xi}$

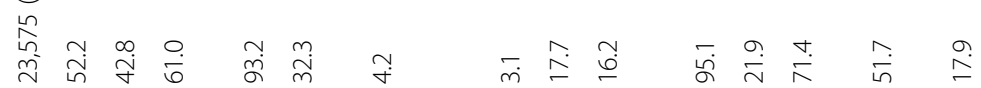

ஓें

$\widehat{r}$
0
0
0
0
0
0
0

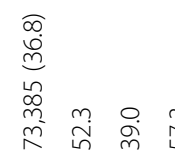

๙ે

文

$\bar{m} \stackrel{n}{\stackrel{n}{=}}$

我

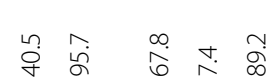

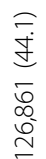

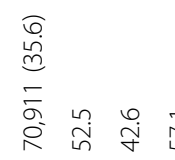

in बं

$\bar{n} \stackrel{n}{\infty} \stackrel{m}{n}$

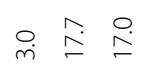

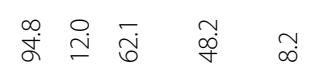

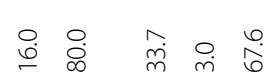

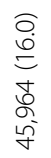

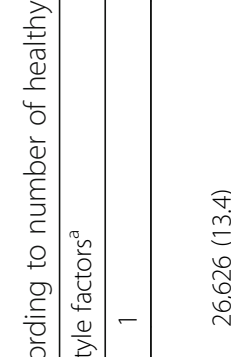

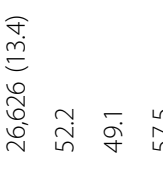

ふָં

$\stackrel{\infty}{0}$

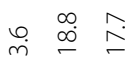

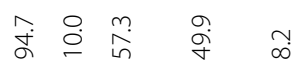

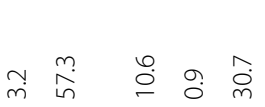

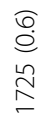

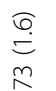

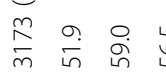

๙ু

$\stackrel{9}{n}$

$\stackrel{n}{m} \stackrel{m}{\infty} \stackrel{\infty}{\infty} \stackrel{\infty}{-}$

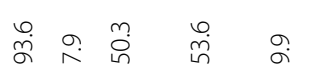

o.
vo

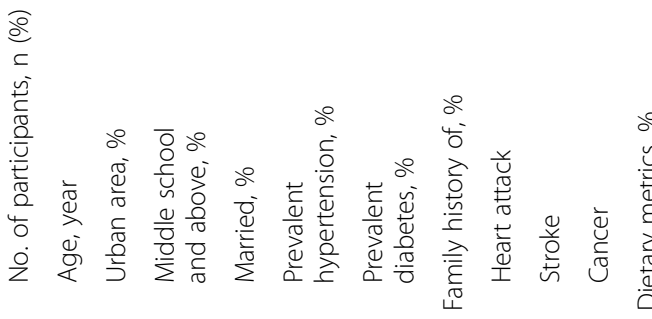

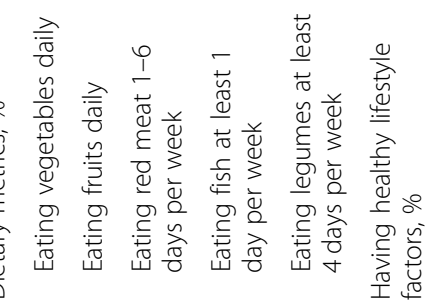

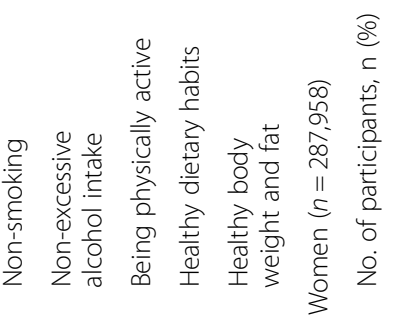




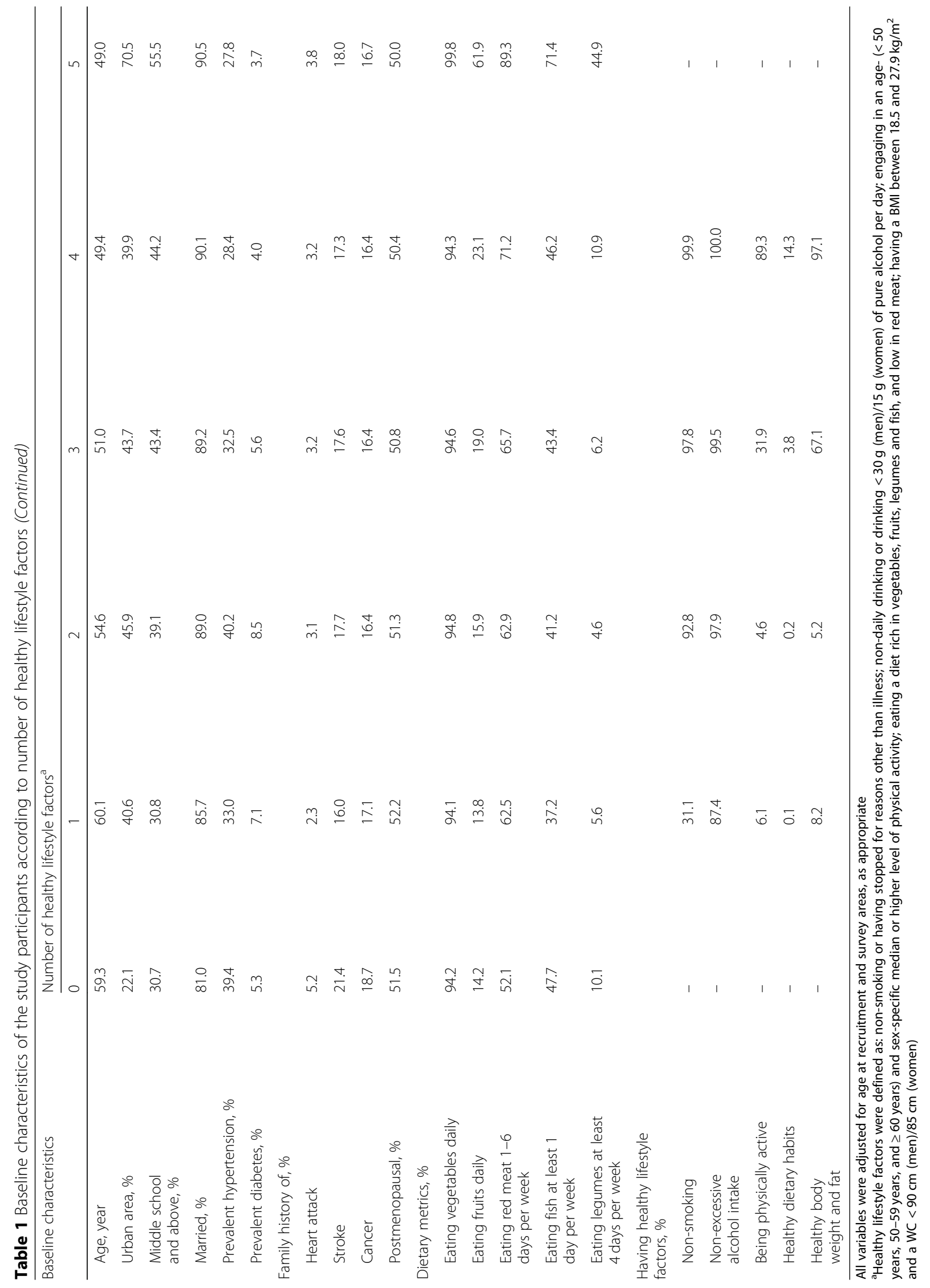




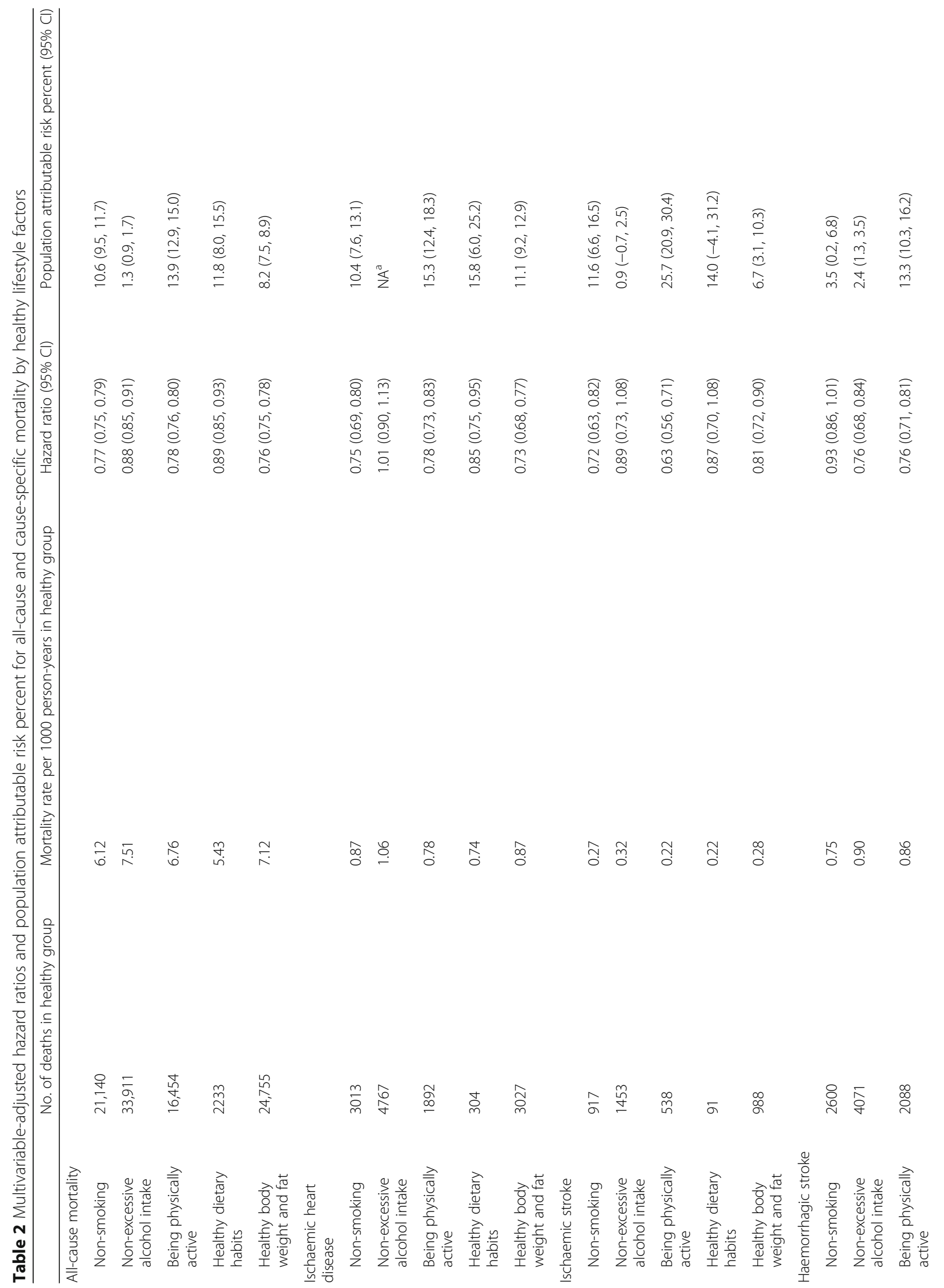




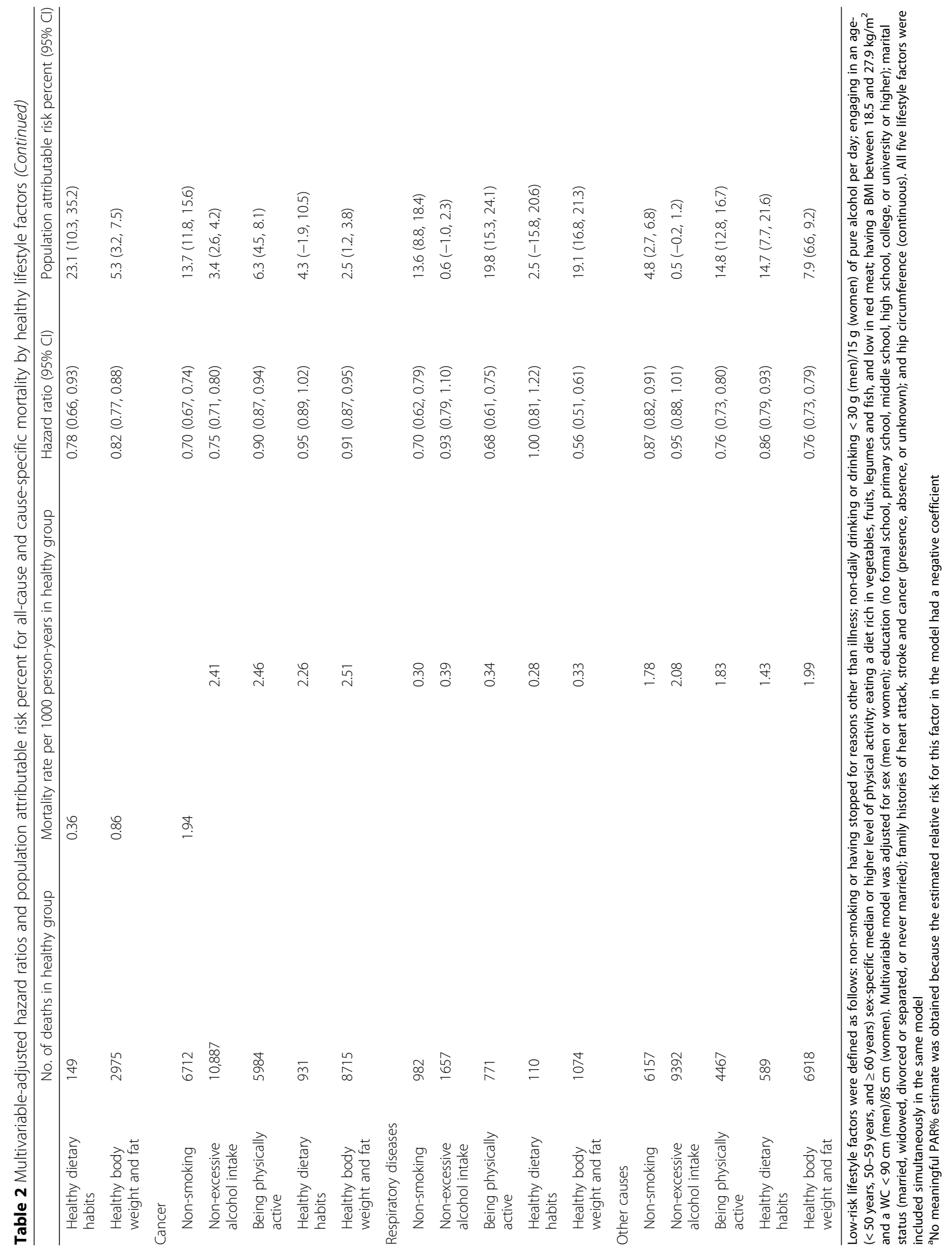




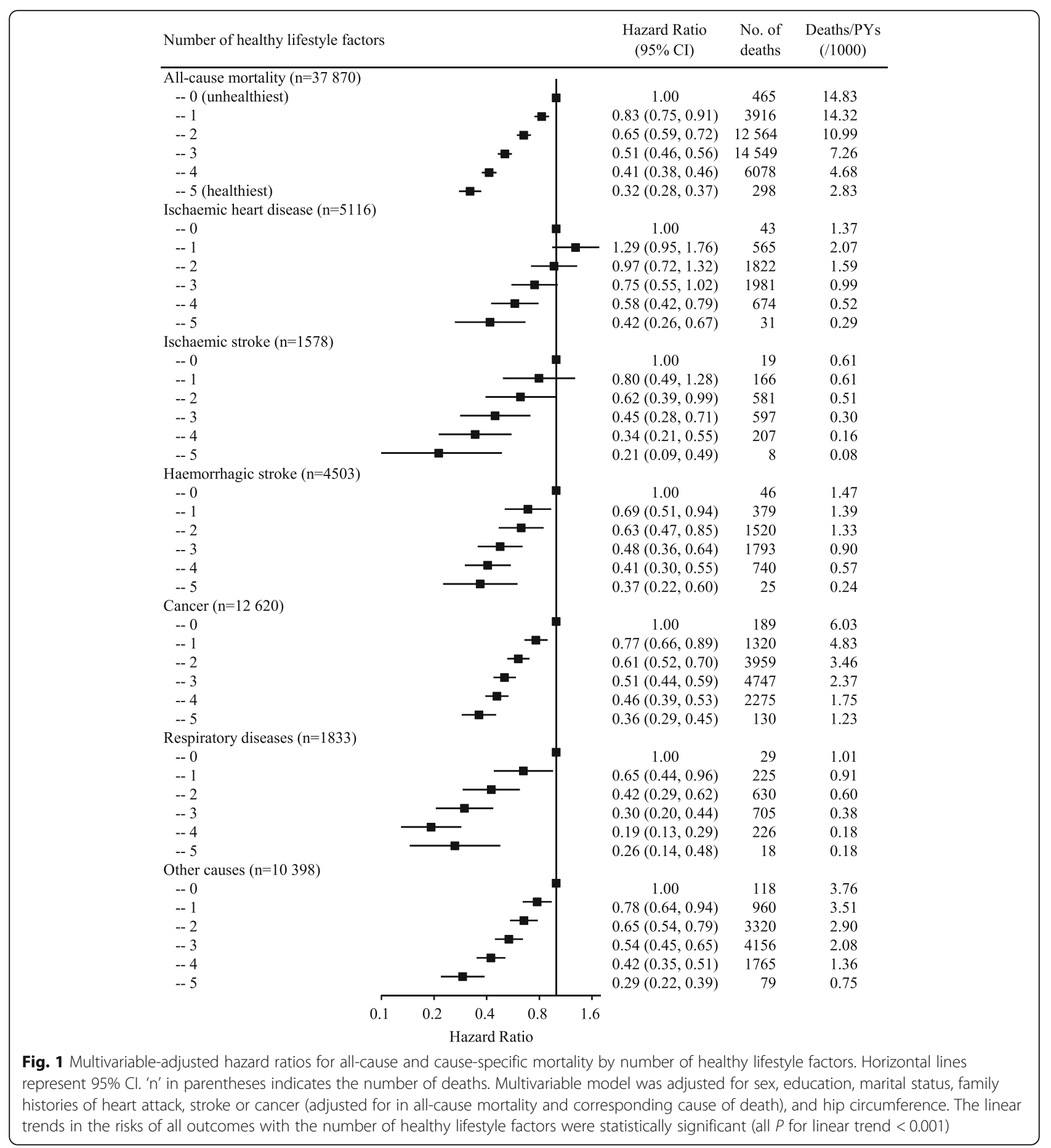

$8.2 \%(7.5,8.9 \%)$ for extreme weight and abdominal obesity (Table 2). The combined PAR\% of all-cause mortality due to smoking, lack of physical activity, and unhealthy diet was $32.2 \%$ (26.8, 37.4\%), which rose up to $37.8 \%(32.4,42.8 \%)$ if additionally considering extreme weight and abdominal obesity. Further inclusion of excessive alcohol intake caused little increase in PAR\% (38.5\%; 95\% CI: 33.0, 43.8\%) (Table 3).
The risk attributable to all these modifiable lifestyle factors varied by specific cause of death, ranging from $26.9 \%(16.7,36.6 \%)$ for cancer to $47.9 \%(22.7,67.0 \%)$ for ischaemic stroke.

In the subgroup analyses, broadly similar PAR\% estimates were obtained from subgroups stratified according to sex, age, education, income, residence, and family history. Nevertheless, the estimated PAR\% of all-cause 
Table 3 Multivariable-adjusted population attributable risk percent for all-cause and cause-specific mortality by specific combination of healthy lifestyle factors

\begin{tabular}{llll}
\hline & $\begin{array}{l}\text { Non-smoking, being physically } \\
\text { active, healthy dietary habits }\end{array}$ & $\begin{array}{l}\text { + Healthy body } \\
\text { weight and fat }\end{array}$ & $\begin{array}{c}\text { + Non-excessive } \\
\text { alcohol intake }\end{array}$ \\
\hline $\begin{array}{l}\text { All-cause mortality } \\
\text { Cause-specific mortality }\end{array}$ & $32.2(26.8,37.4)$ & $37.8(32.4,42.8)$ & $38.5(33.0,43.8)$ \\
$\quad$ Ischaemic heart disease & $36.1(21.8,48.9)$ & $43.2(29.6,55.2)$ & NA \\
$\begin{array}{l}\text { Ischaemic stroke } \\
\text { Haemorrhagic stroke }\end{array}$ & $33.7(19.3,62.9)$ & $47.4(23.0,66.2)$ & $47.9(22.7,67.0)$ \\
Cancer & $22.7(15.9,52.7)$ & $39.1(19.4,55.7)$ & $40.5(20.5,57.3)$ \\
Respiratory diseases & $32.7(7.3,54.2)$ & $24.6(14.6,34.2)$ & $26.9(16.7,36.6)$ \\
Other causes & $30.8(20.2,40.7)$ & $45.4(23.0,63.3)$ & $35.8(22.5,64.1)$ \\
\hline
\end{tabular}

Multivariable model was adjusted for sex, education, marital status, family histories of heart attack, stroke or cancer (adjusted for in all-cause mortality and corresponding cause of death), and hip circumference. All five lifestyle factors were included simultaneously in the same model

${ }^{a}$ The PAR\% of ischaemic heart disease for 5 factors was not available because the estimated relative risk for excessive alcohol intake had a negative coefficient

mortality for five factors among participants with prevalent hypertension and/or diabetes at baseline was $41.1 \%$ (34.1, 47.5\%), higher than those without both (32.7\%; 95\% CI: 23.3, 41.6\%) (Additional file 1: Table S5). Repeated analysis performed among never-regular smokers exhibited lower estimates for ischaemic stroke and cancer than those obtained from the whole cohort (Additional file 1: Table S6). To further rule out the potential reverse causality, we separately excluded underweight participants and deaths that occurred in the first 2 years of follow-up, both of which did not lead to remarkable changes in PAR\% estimates.

\section{Discussion}

In this large, nationwide, prospective cohort study of Chinese adults, each of the five predefined healthy lifestyle factors was independently associated with lower risk of all-cause mortality. In combination, the total mortality risk for participants who had five healthy factors was significantly lower, compared with their counterparts in the unhealthiest group. Assuming causal relationships existed, nearly two-fifths of total deaths in this population during 10 years of follow-up, including over two-fifths of deaths from major CVDs and respiratory diseases, one quarter of deaths from cancer, and one-third of deaths from other causes, could have been prevented through lifestyle modification. The proportion of theoretically preventable deaths was even higher among participants with hypertension and/or diabetes.

Consistent with previous studies [4-6, 8-14], our findings indicated a significant inverse relationship between the number of healthy lifestyle factors and all-cause mortality. However, mortality burden attributable to an unhealthy lifestyle varied across studies. Pooled analysis of the Nurses' Health Study and the Health Professionals Follow-up Study showed that the PAR\% of nonadherence to never smoking, moderate alcohol intake, moderate to vigorous physical activity, a healthy dietary pattern, and an optimal body weight was $60.7 \%$ (53.6, 66.7\%) for all-cause mortality [14]. Similar considerable proportional reduction in all-cause mortality was estimated in British and European cohorts $[8,11]$. However, findings from the Asian population exhibited lower estimates for all-cause mortality, about $30 \%$ in women and $45 \%$ in men [30-33], which approximated to our results. Proposed explanations for this Asian-white difference included differences in lifestyle and genetic factors. In addition, infection, occupational and environmental exposure are still major causes of disease burden in low- and middle-income countries.

In the present study, the mortality burden attributable to an unhealthy lifestyle was higher for CVD than for cancer, which is in line with most previous findings [11, $12,14,32]$. It was estimated that the PAR\% of nonadherence to 5 healthy lifestyle factors was $71.7 \%$ (58.1, 81.0\%) for CVD mortality in the US population, in contrast to $51.7 \%$ (37.1, 62.9\%) for cancer mortality [14]. These lifestyle factors common to CVD and cancer, however, yielded different magnitude of effects on each type of disease. Environmental carcinogens and some infectious agents, as key underlying causes for cancer, may also result in the lower PAR\% estimate for cancer mortality.

Few studies have addressed the associations between a combination of lifestyle factors and stroke mortality. The Singapore Chinese Health Study observed 75\% lower risk of cerebrovascular disease mortality among individuals with $\geq 5$ protective lifestyle factors [34]. Findings from the Japan Collaborative Cohort Study showed the PAR\% was 45.0 and $43.4 \%$ in men and women, respectively [35]. Due to limited sample sizes, analyses were not performed separately for ischaemic and haemorrhagic stroke in these two studies. Our study which further identified stroke subtypes for the first time showed that despite the 
differences in pathogenesis, the proportion of deaths attributable to an unhealthy lifestyle were generally similar between ischaemic stroke and haemorrhagic stroke.

Earlier studies showed a healthy lifestyle also protected against non-CVD non-cancer mortality [8, 10-12]. To our knowledge, this is the first study to investigate the combined effects of lifestyle factors on respiratory disease mortality, one of the major contributors to the global burden of disease. Nearly half of the deaths from respiratory diseases might have been prevented through adopting a healthy lifestyle in the present Chinese population. Also, in the present study, infectious diseases and external causes (e.g., injury) accounted for one-third of the deaths from other causes. The strong gradient for the lower risk of mortality from other causes suggested the favourable effects of a healthy lifestyle may be not limited to NCDs.

Of note, the healthy group for alcohol intake comprised only moderate drinkers in some previous studies $[5,8,10,12,14,34]$, while the present analyses included both non-daily drinkers and moderate drinkers. The application of different definitions would generate distinctive estimates of alcohol-attributable burden. Yet, our data showed that the cardioprotective effect of light to moderate alcohol intake was counterbalanced by higher risk of cancer mortality. Recently, the Global Burden of Disease Study 2016 reexamined the complex association of alcohol intake with health and concluded that consuming zero standard drinks daily minimized the overall health risk [36], pointing to a need to revisit current alcohol control policies. Given the relatively lower prevalence of regular drinking in the Chinese population, especially among women, encouraging moderate intake of alcohol for abstainers would have an extensive and uncertain influence. Careful deliberations are required when making recommendations about alcohol intake.

The chief strengths of our study include its prospective design, large sample size and a large number of accumulated death cases, which enabled us to comprehensively assess the relationships between a combination of lifestyle factors and multiple death outcomes. The inclusion of a geographically dispersed and socioeconomically diverse population permitted us to examine the associations across several important subgroups such as residence, sex, age and education, which makes our results more informative.

This study was also subject to several limitations. Causal inference with observational data is susceptible to several possible sources of bias. Some measurement errors were inevitable since the lifestyle behaviours were self-reported. No separate validation of the questions on physical activity by comparing directly with a reference method has been undertaken; however, these questions were adapted from validated questionnaires used in several other studies, with some additional modifications after a pilot study. Such misclassification should be nondifferential in prospective studies and tends to attenuate the associations. The present analyses only used information on lifestyle factors collected at baseline, and could not necessarily account for the impact of longterm lifestyle patterns. We excluded participants with prior major chronic diseases, and participants who might be in poor health conditions manifested by underweight and dying soon after the start of follow-up. Though carefully dealt with, reverse causality may be still present. Besides, residual confounding due to unmeasured or unknown factors cannot be completely ruled out in observational research. In developing countries including China, physical activity is derived mainly from occupation and housework [37], which together made up nearly $90 \%$ of daily physical activity in the CKB population [38]. Therefore, we defined the healthy group using the total daily physical activity level, instead of the goal levels recommended by current guidelines which were based on leisure-time physical activity. Detailed dietary information was unavailable, and consequently the association between dietary factors and mortality may be underestimated.

\section{Conclusions}

This large prospective cohort study of Chinese adults confirmed that a substantial reduction in the burden of cardiovascular, respiratory diseases and cancer could be achieved by adherence to a healthy lifestyle pattern. If all participants had followed this lifestyle pattern, approximately two-fifths of total deaths during 10 years of follow-up could have been prevented. In light of rapid population aging and constrained medical resources in China and other developing countries, cost-effective population-wide lifestyle interventions that are affordable for most countries could be a better way to respond to the challenges posed by NCDs.

\section{Supplementary information}

Supplementary information accompanies this paper at https://doi.org/10. 1186/s12966-019-0860-z.

Additional file 1: Supplementary material and tables.

\section{Abbreviations}

BMI: Body mass index; Cl: Confidence interval; CKB: China Kadoorie Biobank; COPD: Chronic obstructive pulmonary disease; CVD: Cardiovascular diseases; HR: Hazard ratio; IHD: Ischaemic heart disease; MET: Metabolic equivalent task; NCD: Non-communicable disease; PAR\%: Population attributable risk percent; WC: Waist circumference

\section{Acknowledgments}

The most important acknowledgment is to the participants in the study and the members of the survey teams in each of the 10 regional centres, as well as to the project development and management teams based at Beijing,

Oxford and the 10 regional centres. 


\section{Authors' contributions}

$J \mathrm{~L}$ and $\mathrm{LL}$ conceived and designed the study. $\mathrm{LL}, \mathrm{ZC}$, and $\mathrm{JC}$, as the members of CKB steering committee, designed and supervised the conduct of the whole study, obtained funding, and together with YG, ZB, CY, LY, YC, $H D, H L$ and $F L$ acquired the data. $N Z$ and $Y H$ analysed the data. NZ drafted the manuscript. $J L$ and $L L$ contributed to the interpretation of the results and critical revision of the manuscript for important intellectual content and approved the final version of the manuscript. All authors reviewed and approved the final manuscript. $J L$ and $L L$ are the guarantors.

\section{Funding}

This work was supported by National Natural Science Foundation of China (91846303) and grants (2016YFC0900500, 2016YFC0900501, 2016YFC0900504, 2016YFC1303904) from the National Key R\&D Program of China. The CKB baseline survey and the first re-survey were supported by a grant from the Kadoorie Charitable Foundation in Hong Kong. The long-term follow-up is supported by grants from the UK Wellcome Trust (202922/Z/16/Z, 088158/Z/ 09/Z, 104085/Z/14/Z), National Natural Science Foundation of China (81390540, 81390544, 81390541), and Chinese Ministry of Science and Technology (2011BAI09B01). The funders had no role in the study design, data collection, data analysis and interpretation, writing of the report, or the decision to submit the article for publication.

\section{Availability of data and materials}

The dataset supporting the conclusions of this article is available from the study website (http://www.ckbiobank.org), along with the access policy and procedures.

\section{Ethics approval and consent to participate}

The study protocol was approved by the Ethics Review Committee of the Chinese Centre for Disease Control and Prevention (Beijing, China: 005/2004) and the Oxford Tropical Research Ethics Committee, University of Oxford (UK: 025-04). All participants provided written informed consent before taking part in the study.

\section{Consent for publication}

Not applicable.

\section{Competing interests}

The authors declare that they have no competing interests.

\section{Author details}

'Department of Epidemiology and Biostatistics, School of Public Health, Peking University Health Science Centre, 38 Xueyuan Road, Beijing 100191, China. ${ }^{2}$ Chinese Academy of Medical Sciences, Beijing, China. ${ }^{3}$ Medical Research Council Population Health Research Unit at the University of Oxford, Oxford, UK. ${ }^{4}$ Clinical Trial Service Unit \& Epidemiological Studies Unit (CTSU), Nuffield Department of Population Health, University of Oxford, Oxford, UK. ${ }^{5}$ NCDs Prevention and Control Department, Meilan Centre for Disease Control and Prevention, Haikou, Hainan, China. ${ }^{6}$ Suzhou Centre for Disease Control and Prevention, Suzhou, Jiangsu, China. ${ }^{7}$ China National Centre for Food Safety Risk Assessment, Beijing, China. ${ }^{8}$ Key Laboratory of Molecular Cardiovascular Sciences (Peking University), Ministry of Education, Beijing, China. ${ }^{9}$ Peking University Institute of Environmental Medicine, Beijing, China.

Received: 5 May 2019 Accepted: 14 October 2019

Published online: 04 November 2019

\section{References}

1. World Health Organization. Noncommunicable diseases country profiles 2018. Geneva: World Health Organization; 2018.

2. Zhou M, Wang H, Zhu J, Chen W, Wang L, Liu S, et al. Cause-specific mortality for 240 causes in China during 1990-2013: a systematic subnational analysis for the global burden of disease study 2013. Lancet. 2016;387(10015):251-72.

3. Bloom DE, Cafiero ET, Jané-Llopis E, Abrahams-Gessel S, Bloom LR, Fathima $S$, et al. The global economic burden of noncommunicable diseases. Geneva: World Economic Forum; 2011.
4. van den Brandt PA. The impact of a Mediterranean diet and healthy lifestyle on premature mortality in men and women. Am J Clin Nutr. 2011;94(3):91320.

5. Carlsson AC, Wandell PE, Gigante B, Leander K, Hellenius ML, de Faire U. Seven modifiable lifestyle factors predict reduced risk for ischemic cardiovascular disease and all-cause mortality regardless of body mass index: a cohort study. Int J Cardiol. 2013;168(2):946-52.

6. Ding D, Rogers K, van der Ploeg H, Stamatakis E, Bauman AE. Traditional and emerging lifestyle risk behaviors and all-cause mortality in middle-aged and older adults: evidence from a large population-based Australian cohort. PLoS Med. 2015;12(12):e1001917.

7. Loef $\mathrm{M}$, Walach $\mathrm{H}$. The combined effects of healthy lifestyle behaviors on all cause mortality: a systematic review and meta-analysis. Prev Med. 2012; 55(3):163-70.

8. Knoops KT, de Groot LC, Kromhout D, Perrin AE, Moreiras-Varela O, Menotti $A$, et al. Mediterranean diet, lifestyle factors, and 10-year mortality in elderly European men and women: the HALE project. JAMA. 2004;292(12):1433-9.

9. Emberson JR, Whincup PH, Morris RW, Wannamethee SG, Shaper AG. Lifestyle and cardiovascular disease in middle-aged British men: the effect of adjusting for within-person variation. Eur Heart J. 2005;26(17):1774-82.

10. Khaw KT, Wareham N, Bingham S, Welch A, Luben R, Day N. Combined impact of health behaviours and mortality in men and women: the EPICNorfolk prospective population study. PLoS Med. 2008;5(1):e12.

11. Kvaavik E, Batty GD, Ursin G, Huxley R, Gale CR. Influence of individual and combined health behaviors on total and cause-specific mortality in men and women: the United Kingdom health and lifestyle survey. Arch Intern Med. 2010;170(8):711-8.

12. Ford ES, Zhao G, Tsai J, Li C. Low-risk lifestyle behaviors and all-cause mortality: findings from the National Health and nutrition examination survey III mortality study. Am J Public Health. 2011;101(10):1922-9.

13. Petersen KE, Johnsen NF, Olsen A, Albieri V, Olsen LK, Dragsted LO, et al. The combined impact of adherence to five lifestyle factors on all-cause, cancer and cardiovascular mortality: a prospective cohort study among Danish men and women. Br J Nutr. 2015;113(5):849-58.

14. Li Y, Pan A, Wang DD, Liu X, Dhana K, Franco OH, et al. Impact of healthy lifestyle factors on life expectancies in the US population. Circulation. 2018; 138(4):345-55.

15. Tsai CF, Thomas B, Sudlow CL. Epidemiology of stroke and its subtypes in Chinese vs white populations: a systematic review. Neurology. 2013;81(3):264-72.

16. Chen Z, Lee L, Chen J, Collins R, Wu F, Guo Y, et al. Cohort profile: the Kadoorie study of chronic disease in China (KSCDC). Int J Epidemiol. 2005; 34(6):1243-9.

17. Chen Z, Chen J, Collins R, Guo Y, Peto R, Wu F, et al. China Kadoorie biobank of 0.5 million people: survey methods, baseline characteristics and long-term follow-up. Int J Epidemiol. 2011;40(6):1652-66.

18. Kurmi OP, Li L, Smith M, Augustyn M, Chen J, Collins R, et al. Regional variations in the prevalence and misdiagnosis of air flow obstruction in China: baseline results from a prospective cohort of the China Kadoorie biobank (CKB). BMJ Open Respir Res. 2014;1 (1):e000025.

19. Du H, Bennett D, Li L, Whitlock G, Guo Y, Collins R, et al. Physical activity and sedentary leisure time and their associations with BMl, waist circumference, and percentage body fat in 0.5 million adults: the China Kadoorie biobank study. Am J Clin Nutr. 2013;97(3):487-96.

20. Lv J, Yu C, Guo Y, Bian Z, Yang L, Chen Y, et al. Adherence to healthy lifestyle and cardiovascular diseases in the Chinese population. J Am Coll Cardiol. 2017;69(9):1116-25.

21. LV J, Yu C, Guo Y, Bian Z, Yang L, Chen Y, et al. Adherence to a healthy lifestyle and the risk of type 2 diabetes in Chinese adults. Int J Epidemiol. 2017:46(5):1410-20.

22. Chinese Nutrition Society. The Chinese dietary guidelines. Beijing: People's Medical Publishing House; 2016.

23. National Health and Family Planning Commission of the People's Republic of China. Criteria of weight for adults (WS/T 428-2013). Beijing: Standards Press of China; 2013.

24. Van Horn L, Carson JA, Appel $\amalg$, Burke LE, Economos C, Karmally W, et al. Recommended dietary pattern to achieve adherence to the American Heart Association/American College of Cardiology (AHA/ACC) guidelines: a scientific statement from the American Heart Association. Circulation. 2016; 134(22):e505-e29.

25. Lloyd-Jones DM, Hong Y, Labarthe D, Mozaffarian D, Appel L, Van Horn L, et al. Defining and setting national goals for cardiovascular health 
promotion and disease reduction: the American Heart Association's strategic impact goal through 2020 and beyond. Circulation. 2010;121(4): 586-613.

26. Yang G, Hu J, Rao KQ, Ma J, Rao C, Lopez AD. Mortality registration and surveillance in China: history, current situation and challenges. Popul Health Metrics. 2005;3(1):3.

27. Liu S, Wu X, Lopez AD, Wang L, Cai Y, Page A, et al. An integrated national mortality surveillance system for death registration and mortality surveillance, China. Bull World Health Organ. 2016;94(1):46-57.

28. Cameron AJ, Magliano DJ, Soderberg S. A systematic review of the impact of including both waist and hip circumference in risk models for cardiovascular diseases, diabetes and mortality. Obes Rev. 2013;14(1):86-94.

29. Spiegelman D, Hertzmark E, Wand HC. Point and interval estimates of partial population attributable risks in cohort studies: examples and software. Cancer Causes Control. 2007;18(5):571-9.

30. Tamakoshi A, Tamakoshi K, Lin Y, Yagyu K, Kikuchi S, Group JS. Healthy lifestyle and preventable death: findings from the Japan collaborative cohort (JACC) study. Prev Med. 2009;48(5):486-92.

31. Yun JE, Won S, Kimm H, Jee SH. Effects of a combined lifestyle score on 10year mortality in Korean men and women: a prospective cohort study. BMC Public Health. 2012;12:673.

32. Nechuta SJ, Shu XO, Li HL, Yang G, Xiang YB, Cai H, et al. Combined impact of lifestyle-related factors on total and cause-specific mortality among Chinese women: prospective cohort study. PLoS Med. 2010;7(9):e1000339.

33. Zhang QL, Zhao LG, Zhang W, Li HL, Gao J, Han LH, et al. Combined impact of known lifestyle factors on Total and cause-specific mortality among Chinese men: a prospective cohort study. Sci Rep. 2017;7(1):5293.

34. Odegaard AO, Koh WP, Gross MD, Yuan JM, Pereira MA. Combined lifestyle factors and cardiovascular disease mortality in Chinese men and women: the Singapore Chinese health study. Circulation. 2011;124(25):2847-54.

35. Eguchi E, Iso H, Tanabe N, Wada Y, Yatsuya H, Kikuchi S, et al. Healthy lifestyle behaviours and cardiovascular mortality among Japanese men and women: the Japan collaborative cohort study. Eur Heart J. 2012;33(4):467-77.

36. Alcohol Collaborators GBD. Alcohol use and burden for 195 countries and territories, 1990-2016: a systematic analysis for the global burden of disease study 2016. Lancet. 2018;392(10152):1015-35.

37. Ng SW, Howard AG, Wang HJ, Su C, Zhang B. The physical activity transition among adults in China: 1991-2011. Obes Rev. 2014;15(Suppl 1):27-36.

38. Du H, Li L, Whitlock G, Bennett D, Guo Y, Bian Z, et al. Patterns and sociodemographic correlates of domain-specific physical activities and their associations with adiposity in the China Kadoorie biobank study. BMC Public Health. 2014;14:826.

\section{Publisher's Note}

Springer Nature remains neutral with regard to jurisdictional claims in published maps and institutional affiliations.

Ready to submit your research? Choose BMC and benefit from:

- fast, convenient online submission

- thorough peer review by experienced researchers in your field

- rapid publication on acceptance

- support for research data, including large and complex data types

- gold Open Access which fosters wider collaboration and increased citations

- maximum visibility for your research: over $100 \mathrm{M}$ website views per year

At BMC, research is always in progress.

Learn more biomedcentral.com/submissions 